\title{
The effects of once-weekly teriparatide on hip structure and biomechanical properties assessed by $\mathrm{CT}$
}

\author{
M. Ito • R. Oishi • M. Fukunaga • T. Sone • T. Sugimoto • \\ M. Shiraki • Y. Nishizawa • T. Nakamura
}

Received: 23 May 2013 / Accepted: 19 August 2013 /Published online: 18 December 2013

(C) The Author(s) 2013. This article is published with open access at Springerlink.com

\begin{abstract}
Summary Once-weekly administration of $56.5 \mu \mathrm{g}$ teriparatide improved cortical bone parameters and biomechanical parameters at the proximal femur by CT geometry analysis.

Introduction The aim of this study was to evaluate the effects of weekly administration of teriparatide [human PTH (1-34)] on bone geometry, volumetric bone mineral density (vBMD), and parameters of bone strength at the proximal femur which were longitudinally investigated using computed tomography $(\mathrm{CT})$.
\end{abstract}

\footnotetext{
M. Ito $(\square)$

Medical Work-Life-Balance Center, Nagasaki University Hospital,

1-7-1 Sakamoto, Nagasaki 852-8501, Japan

e-mail: masako@nagasaki-u.ac.jp

R. Oishi

Clinical Development Center, Asahi Kasei Pharma Corporation,

Tokyo, Japan

M. Fukunaga

Kawasaki Medical School, Okayama, Japan

T. Sone

Department of Nuclear Medicine, Kawasaki Medical School,

Okayama, Japan

T. Sugimoto

Internal Medicine 1, Shimane University Faculty of Medicine,

Shimane, Japan

M. Shiraki

Research Institute and Practice for Involutional Diseases,

Nagano, Japan

Y. Nishizawa

Osaka City University, Osaka, Japan

T. Nakamura

National Center for Global Health and Medicine, Tokyo, Japan
}

Methods The subjects were a subgroup of a recent, randomly assigned, double-blind study (578 subjects) comparing the anti-fracture efficacy of a once-weekly subcutaneous injection of $56.5 \mu \mathrm{g}$ teriparatide with placebo (TOWER trial).

Results Sixty-six ambulatory postmenopausal women with osteoporosis were enrolled at 15 study sites having multidetector row $\mathrm{CT}$, and included women injected with teriparatide ( $n=29,74.2 \pm 5.1$ years) or with placebo $(n=37$, $74.8 \pm 5.3$ years). CT data were obtained at baseline and follow-up scans were performed at 48 and 72 weeks. The data were analyzed to obtain cross-sectional densitometric, geometric, and biomechanical parameters including the section modulus (SM) and buckling ratio (BR) of the femoral neck, inter-trochanter, and femoral shaft. We found that onceweekly teriparatide increased cortical thickness/crosssectional area (CSA) and total area, and improved biomechanical properties (i.e., decreasing BR) at the femoral neck and shaft. Teriparatide did not change the cortical perimeter. Conclusions Our longitudinal analysis of proximal femur geometry by $\mathrm{CT}$ revealed that once-weekly administration of $56.5 \mu \mathrm{g}$ teriparatide improved cortical bone parameters at the femoral neck and shaft and also improved biomechanical parameters.

Keywords Once-weekly injection · Osteoporosis · Proximal femur geometry · Quantitative computed tomography ·

Teriparatide

\section{Introduction}

Parathyroid hormone (PTH) stimulates bone formation and resorption and can increase or decrease bone mass, depending on the dose and timing of administration. Continuous infusions and daily subcutaneous injections of teriparatide stimulate bone formation but have distinct effects on bone 
resorption and bone mass [1,2]. Daily injections of 20 and $40 \mu \mathrm{g}$ teriparatide increased the bone mineral density (BMD) at the lumbar spine by 9 and $13 \%$, and reduced the risk of incident vertebral fractures by 65 and $69 \%$ as relative risk reduction, respectively, as compared with placebo [3].

Weekly injections of $56.5 \mu \mathrm{g}$ teriparatide have been shown to increase BMD at the lumbar spine by $8.1 \%$ after 48 weeks of treatment as determined by dual energy X-ray absorptiometry (DXA) [4]. Anti-fracture efficacy of once-weekly subcutaneous injection of $56.5 \mu \mathrm{g}$ teriparatide for 72 weeks was evaluated in 578 postmenopausal women and older men with primary osteoporosis by a randomized controlled trial, the Teriparatide Once-Weekly Efficacy Research (TOWER) trial [5]. Vertebral fracture risk was reduced by $80 \%$ as relative risk reduction.

Daily treatment with teriparatide reduced the risk of nonvertebral fractures by 35 to $40 \%$ at the 20 and $40 \mu \mathrm{g}$ dose, respectively, and reduced the risk of non-vertebral fragility fractures by 53 and $54 \%$, respectively [3]. Weekly treatment with teriparatide reduced the risk of clinical fragility fractures include non-vertebra by $67 \%$ [5].

The bone geometry in the proximal femur is thought to be strongly related to bone strength, and our previous studies showed that proximal femur geometrical parameters could predict the incidence of neck fracture or inter-trochanter fracture [6].

The reason for reduced risk of non-vertebral fracture may be explained by changes in structure and biomechanical properties by teriparatide treatment. Therefore, it is important to evaluate changes in structure and mechanical properties in each treatment regimen of teriparatide compared to the placebo.

As a surrogate endpoint of the TOWER trial, computed tomography (CT) has been applied to evaluate and compare the effects of teriparatide versus placebo on proximal femur, since $\mathrm{CT}$ evaluation is considered to be a suitable cortical bone assessment. The purpose of this study was to investigate the effect of once-weekly injection of $56.5 \mu \mathrm{g}$ teriparatide on bone geometry, volumetric bone density, and bone strength parameters of the proximal femur, using CT.

\section{Methods}

\section{Subjects}

Subjects in this study were a subset of the original TOWER trial [5], and constituted ambulatory female patients with osteoporosis enrolled at 15 study sites equipped with multidetector row $\mathrm{CT}$ (MDCT) to measure hip BMD, bone geometry, and biomechanical indices. All subjects in this study fulfilled the inclusion and exclusion criteria of the original TOWER trial. Subjects with one to five vertebral fractures with low BMD (T-score $\leq-1.67$ ) at either the lumbar spine (L2-L4), femoral neck, total hip, or radius measured by dualenergy X-ray absorptiometry (DXA) or the right second metacarpal bone measured by radiographic absorptiometry were eligible. Subjects with diseases or using drugs affecting bone or calcium metabolism were excluded. The subjects were randomly divided into two groups, either weekly subcutaneous injection of $56.5 \mu \mathrm{g}$ teriparatide or placebo for 72 weeks. All subjects received daily supplements of $610 \mathrm{mg}$ calcium, $400 \mathrm{IU}$ vitamin $\mathrm{D}_{3}$, and $30 \mathrm{mg}$ magnesium.

The original trial was conducted in compliance with the ethical principles stated in the Declaration of Helsinki and Good Clinical Practice. The trial was approved by the institutional review boards at each site and all subjects provided written informed consent before enrollment.

\section{CT data acquisition}

CT data were obtained at baseline and follow-up scans were performed at 48 and 72 weeks of treatment, using the scanning and reconstruction protocol previously described [7]. The scanning conditions (X-ray energy, 120 to $140 \mathrm{kV}$; X-ray current, $250 \mathrm{~mA}$; rotation speed, 0.8 to $1.0 \mathrm{~s} /$ rot; beam pitch, 0.5625 to 0.9375 ) and reconstruction parameters were predefined for each type of CT scanner. Beam pitch is defined as the ratio of table feed per rotation to the collimation, where collimation is the product of slice-thickness and the number of slices in each rotation. Field of View (FOV) was defined as $350 \mathrm{~mm}$ to cover bilateral proximal femur regions. In-plane spatial resolution of 0.625 to $0.652 \mathrm{~mm}$ and reconstructed slice thickness of 0.500 to $0.625 \mathrm{~mm}$ were adjusted according to $\mathrm{CT}$ scanner type. The $\mathrm{CT}$ values were converted to bone mineral scale by using a solid reference phantom, B-MAS200 (Fujirebio Inc., Tokyo, Japan) containing hydroxyapatite (HA) at $0,50,100,150$, and $200 \mathrm{mg} / \mathrm{cm}^{3}$.

The MDCT scanners used in this study originally included four Asteion 4, one Aquilion 16 TSX-101A, one Aquilion 32, and three Aquilion 64 scanners (Toshiba Medical Systems Corporation); two LightSpeed Ultra_16, one LightSpeed VCT_64, and one BrightSpeed Elite_16 scanner (GEYokogawa Medical); and one Somatom 16, and one Somatom 64 scanner (Siemens, AG).

\section{Scanner cross-calibration}

Good linear correlations between the CT values and HA concentrations were demonstrated ( $r=0.993$ to $1.000 ; p<$ 0.0006 to 0.0001 ) in all CT scanners. Differences in CT values according to X-ray energy were corrected using the reference phantom to convert $\mathrm{CT}$ values to $\mathrm{HA}$ equivalent values. However, it was necessary to confirm the longitudinal stability of the CT values of the threshold value used to define the cortical bone. 
Quality assurance (QA) scans with a Type 3 Mindways Phantom (Mindways Software, Austin, TX, USA) were performed before and after study measurements took place at the individual clinical sites in order to adjust for longitudinal changes of the detector. QA measurements were evaluated according to the quantitated computed tomography (QCT)Pro QA Guide from Mindways. There was no drift from baseline to the completion of treatment in any $\mathrm{CT}$ apparatus.

\section{Subject positioning for CT scanning}

Subjects were scanned in the supine position with the reference phantom beneath them and placed so as to cover a region from the top of the acetabulum to $4 \mathrm{~cm}$ below the bottom of the lesser trochanter in each hip joint (average slice number was 298). Buffer material to protect artifact, such as a bolus bag or blanket, were placed between the subject and the CT calibration phantom. The subject's hands and arms were placed over their head or as high on the chest as was comfortable to avoid interfering with the scan area. The CT scanner table height was set to the center of the greater trochanter.

Analysis of BMD, bone geometry, and biomechanical properties obtained by $\mathrm{CT}$

Subject data were evaluated with QCT-Pro software v4.1.3 with the QCT-Pro Bone Investigational Toolkit v2.0 (BIT) (Mindways Software) for the femoral neck, inter-trochanter, and femoral shaft regions. All measurements were analyzed by a radiologist (M. Ito) blinded to treatment-group assignment.

\section{QCT-Pro CTXA proximal femur exam analysis}

The exact 3D rotation of the femur and the threshold setting for defining the bone contours appeared to be the two most critical steps for achieving accuracy and reproducibility in the automated procedures performed by QCT-Pro [7, 8]. The outer cortical margin was defined using uniform HA equivalent BMD values.

The femoral neck axis was identified visually and also automatically with the "Optimize FN Axis" algorithm. Using the eccentricity registration method, a series of 10 reformatted $1-\mathrm{mm}$ slices was positioned perpendicularly to the neck axis. The definitions of inter-trochanter and femoral-shaft cross-section are consistent with the DXAbased hip structure analysis methods developed by Tom Beck [9]. All steps were compared visually across all visits and repeated if the positioning did not appear to be accurate.

The eccentricity registration method was applied to define the volume of interest (VOI) consisting of six reformatted 1$\mathrm{mm}$ slices oriented perpendicular to the neck axis. QCT BIT processing was then performed with a fixed-bone threshold for cortical separation set to $350 \mathrm{mg} / \mathrm{cm}^{3}$ for all subjects and visits. This application was used to measure hip axis length (HAL), femoral neck angle (FNA), and neck width, vBMD, cross-sectional area (CSA), and cross-sectional bone mass of the femoral neck, inter-trochanter, and femoral shaft (total and cortical regions). Cortical thickness and perimeter were also measured. Biomechanical properties were also derived from the cross-sectional parameters of the femoral neck, intertrochanter, and shaft.

Analysis of cross-sectional bone geometry and volumetric BMD

The cross-sectional femoral neck data were based on the geometrical axis to calculate cortical CSA (in square centimeter), total CSA (in square centimeter), volumetric cortical BMD (cortical vBMD; in milligram per cubic centimeter), total volumetric BMD (total vBMD; in milligram per cubic centimeter), total bone mass (in gram), and cortical bone mass (in gram). In this study, total CSA was defined as the estimated total mineralized area. Cortical thickness (in millimeter) and cortical perimeter (in centimeter) were also derived.

\section{Biomechanical parameters}

Because biomechanical parameters were determined on the principal axis, the cross-sectional moment of inertia (CSMI; in millimeters to the fourth power), the section modulus (SM; in cubic millimeter) and the buckling ratio (BR) were calculated from bone density and geometrical data. The CSMI is defined by the integration of the products of incremental CSA and the square of their distance from the center of mass (centroid). The $\mathrm{SM}$ is the ratio of CSMI to the maximal distance of the material from the centroid, which is directly related to strength with respect to a corresponding bending stress. Due to local buckling, failure occurs on the compressive surface, and BR was calculated in this study as the maximal distance from the centroid divided by the average cortical thickness [9].

Reproducibility of the analysis done by the QCT-Pro program was calculated by using five repeated measurements with visual matching each time from CT data sets without visible artifacts from seven healthy subjects. The coefficient of variation, as determined by the root mean square standard deviation divided by the mean, was $1.49 \%$ for total vBMD, $2.63 \%$ for cortical vBMD, $1.12 \%$ for total mass, $1.71 \%$ for total CSA, $2.11 \%$ for cortical CSA, $2.11 \%$ for cortical perimeter, and $3.58 \%$ for cortical thickness at the femoral neck [10].

Statistics

All statistical analyses were performed on subjects who had been randomized and had evaluable observations for QCT 
assessment both at baseline and post-dose (48 or 72 weeks). Paired and Student's $t$ tests, and chi-square test were used and Pearson's correlation coefficients are shown. All $p$ values calculated in the analysis were two-sided and were not adjusted for multiple testing. Statistical analyses were done with SAS version 9.1 (SAS Institute, Cary, USA).

\section{Results}

A total of 66 subjects were enrolled and randomly assigned to two treatment groups. There were 29 in the teriparatide group (age 66 to 83 years; mean \pm SD, $74.2 \pm 5.1$ years) and 37 in the placebo group (age 66 to 88 years, $74.8 \pm 5.3$ years). Table 1 shows the background of subjects and bone characteristics at baseline in both groups. There were no significant differences between the two groups in age, height, weight, body mass index (BMI), years after menopause, BMD at the spine and hip, or the number of vertebral fractures $(p>0.05)$.

\section{Effect of teriparatide on bone geometry parameters}

Baseline and the observed change of bone geometry parameters are shown in Table 2. There were no significant differences at baseline for any bone geometry parameter at the femoral neck, inter-trochanter, and femoral shaft between the teriparatide and placebo groups. Compared to baseline, weekly teriparatide significantly increased cortical thickness at the femoral neck (3.5\%, 48 weeks) and shaft (2.6\%, 72 weeks). Cortical CSA increased at the inter-trochanter $(3.8 \%$, 48 weeks) and femoral shaft (2.7\%, 72 weeks). Total CSA increased at the inter-trochanter $(3.8 \%$ at 48 weeks; $4.7 \%$,

Table 1 Subject baseline demographics and bone characteristics

\begin{tabular}{lll}
\hline & $\begin{array}{l}\text { Teriparatide } \\
(n=29)\end{array}$ & $\begin{array}{l}\text { Placebo } \\
(n=37)\end{array}$ \\
\hline Age (years) & $74.2 \pm 5.1$ & $74.8 \pm 5.3$ \\
Body height (cm) & $147.8 \pm 5.1$ & $147.5 \pm 5.5$ \\
Body weight (kg) & $50.9 \pm 8.4$ & $49.1 \pm 8.5$ \\
Body mass index (BMI) $\left(\mathrm{kg} / \mathrm{m}^{2}\right)$ & $23.3 \pm 3.5$ & $22.5 \pm 3.5$ \\
Years after menopause (years) & $24.6 \pm 6.5$ & $25.2 \pm 6.6$ \\
Bone mineral density (T-score) & & \\
$\quad$ Lumbar spine (L2-4) & $-2.6 \pm 1.0$ & $-2.8 \pm 0.8$ \\
$\quad$ Femoral neck & $-2.4 \pm 0.7$ & $-2.6 \pm 0.7$ \\
$\quad$ Femoral total hip & $-2.0 \pm 1.0$ & $-2.5 \pm 1.2$ \\
Number of prevalent vertebral fractures & $1.6 \pm 1.1$ & $1.3 \pm 1.3$ \\
\hline
\end{tabular}

Bone mineral density was measured by dual X-ray absorptiometry Data are mean \pm SD

Two subjects who were diagnosed with a BMD evaluation at the radius or metacarpal bone in the teriparatide group and one subject evaluated at the metacarpal bone in the placebo group were included
72 weeks) and femoral shaft $(2.5 \%, 72$ weeks). Cortical vBMD decreased at the femoral neck $(1.2 \%, 72$ weeks $)$ and inter-trochanter (1.5\%, 72 weeks). BR was also decreased at the femoral shaft ( $3.3 \%, 72$ weeks). There was no change in cortical perimeter at any site. There were no significant changes observed in the placebo group except for an increase in BR at the inter-trochanter (4.3\%, 48 weeks).

Effect of teriparatide on cortical thickness, cortical and total CSA, and cortical perimeter compared to placebo

Comparisons of cortical thickness, CSA, and perimeter between the two groups are shown in Fig. 1. Significantly higher cortical thickness was observed in the teriparatide group at the femoral neck (48 and 72 weeks) and shaft ( 72 weeks) (Fig. 1a). Significantly higher cortical CSA at the intertrochanter (at 48 weeks) and at the femoral neck (72 weeks) were observed in the teriparatide group (Fig. 1b). Significantly higher total CSA at the inter-trochanter (48 and 72 weeks) and the femoral shaft ( 72 weeks) were observed in the teriparatide group (Fig. 1c). No significant differences were observed in the cortical perimeters between the teriparatide and placebo groups at any measurement site (Fig. 1d).

In summary, both cortical thickness and CSA increased in all three regions following treatment with teriparatide and decreased in the placebo group. In contrast, there was no change in cortical perimeter following once-weekly injections of teriparatide.

Effect of teriparatide on cortical and total vBMD compared to placebo

The comparison of cortical and total vBMD between the teriparatide and placebo groups is shown in Fig. 2. No significant differences in cortical vBMD were observed between the groups. A significant higher total vBMD in the teriparatide group was observed at the inter-trochanter (Fig. 2b).

Effect of teriparatide on biomechanical parameters compared to placebo

The differences in biomechanical parameters are shown in Fig. 3. SM changes in the teriparatide group at the three measurement sites were positive but not significant (Fig. 3a). BR values in the teriparatide group at the femoral neck (48 and 72 weeks) and shaft (72 weeks) were significantly lower compared to placebo (Fig. 3b).

Relationship between changes in cortical thickness and other parameters

In order to understand the relationships between the parameters, the correlations between the percent changes in cortical 
Table 2 Baseline QCT measurements and the percent changes at 48 and 72 weeks

\begin{tabular}{|c|c|c|c|c|c|c|c|}
\hline \multirow[t]{3}{*}{ Site } & \multirow[t]{3}{*}{ Parameter } & \multicolumn{3}{|l|}{ Teriparatide } & \multicolumn{3}{|l|}{ Placebo } \\
\hline & & \multicolumn{3}{|l|}{$(n=29)$} & \multicolumn{3}{|l|}{$(n=37)$} \\
\hline & & Baseline & 48 weeks & 72 weeks & Baseline & 48 weeks & 72 weeks \\
\hline \multirow[t]{8}{*}{ Femoral neck } & Cortical thickness (mm) & $1.47 \pm 0.24$ & $3.5 \pm 7.1^{*}$ & $3.6 \pm 9.0$ & $1.52 \pm 0.26$ & $-0.5 \pm 6.8$ & $-0.9 \pm 5.1$ \\
\hline & Cortical CSA $\left(\mathrm{cm}^{2}\right)$ & $0.86 \pm 0.15$ & $2.8 \pm 7.6$ & $2.2 \pm 7.9$ & $0.90 \pm 0.15$ & $-0.6 \pm 6.1$ & $0.0 \pm 5.2$ \\
\hline & Total CSA $\left(\mathrm{cm}^{2}\right)$ & $1.22 \pm 0.21$ & $2.2 \pm 7.1$ & $3.2 \pm 7.3$ & $1.28 \pm 0.19$ & $-0.2 \pm 5.1$ & $0.6 \pm 4.8$ \\
\hline & Cortical perimeter $(\mathrm{cm})$ & $10.96 \pm 0.97$ & $-1.6 \pm 4.4$ & $-1.4 \pm 5.9$ & $10.96 \pm 0.93$ & $0.2 \pm 3.8$ & $0.1 \pm 3.5$ \\
\hline & Cortical vBMD $\left(\mathrm{mg} / \mathrm{cm}^{3}\right)$ & $667.00 \pm 52.57$ & $-0.6 \pm 2.7$ & $-1.2 \pm 2.3^{*}$ & $676.84 \pm 46.65$ & $-0.2 \pm 4.3$ & $-0.8 \pm 3.1$ \\
\hline & Total vBMD $\left(\mathrm{mg} / \mathrm{cm}^{3}\right)$ & $221.77 \pm 31.77$ & $1.0 \pm 3.4$ & $0.0 \pm 3.8$ & $227.98 \pm 35.35$ & $-0.7 \pm 4.4$ & $-1.2 \pm 3.3$ \\
\hline & $\mathrm{SM}\left(\mathrm{cm}^{3}\right)$ & $0.38 \pm 0.1$ & $3.4 \pm 8.2$ & $2.3 \pm 8.8$ & $0.38 \pm 0.1$ & $-0.3 \pm 8.2$ & $0.6 \pm 7.5$ \\
\hline & $\mathrm{BR}$ & $13.96 \pm 2.32$ & $-3.2 \pm 7.4$ & $-3.0 \pm 8.3$ & $13.44 \pm 3.22$ & $1.3 \pm 6.2$ & $1.8 \pm 6.1$ \\
\hline \multirow[t]{8}{*}{ Inter-trochanter } & Cortical thickness (mm) & $1.43 \pm 0.26$ & $0.9 \pm 5.9$ & $0.7 \pm 6.4$ & $1.51 \pm 0.29$ & $-2.3 \pm 6.6$ & $-0.8 \pm 7.7$ \\
\hline & Cortical CSA $\left(\mathrm{cm}^{2}\right)$ & $1.38 \pm 0.29$ & $3.8 \pm 7.4^{*}$ & $2.9 \pm 8.6$ & $1.54 \pm 0.33$ & $-1.6 \pm 5.6$ & $-0.6 \pm 5.5$ \\
\hline & Total CSA $\left(\mathrm{cm}^{2}\right)$ & $2.38 \pm 0.45$ & $3.8 \pm 8.8^{*}$ & $4.7 \pm 9.4^{*}$ & $2.59 \pm 0.5$ & $-1.8 \pm 5.6$ & $-0.6 \pm 4.8$ \\
\hline & Cortical perimeter $(\mathrm{cm})$ & $16.76 \pm 1.15$ & $0.2 \pm 3.3$ & $-0.6 \pm 2.0$ & $17.12 \pm 1.18$ & $0.6 \pm 2.4$ & $0.0 \pm 2.1$ \\
\hline & Cortical vBMD $\left(\mathrm{mg} / \mathrm{cm}^{3}\right)$ & $638.96 \pm 48.01$ & $-0.4 \pm 2.4$ & $-1.5 \pm 2.1^{* *}$ & $646.03 \pm 44.09$ & $-0.3 \pm 2.9$ & $-0.6 \pm 2.4$ \\
\hline & Total vBMD $\left(\mathrm{mg} / \mathrm{cm}^{3}\right)$ & $186.13 \pm 35.97$ & $1.1 \pm 3.3$ & $0.7 \pm 4.7$ & $196.1 \pm 35.7$ & $-1.5 \pm 4.5$ & $-1.5 \pm 4.8$ \\
\hline & $\mathrm{SM}\left(\mathrm{cm}^{3}\right)$ & $0.67 \pm 0.18$ & $5.0 \pm 15.8$ & $4.1 \pm 11.8$ & $0.73 \pm 0.18$ & $2.4 \pm 12.0$ & $1.8 \pm 10.2$ \\
\hline & $\mathrm{BR}$ & $19.71 \pm 3.6$ & $2.1 \pm 10.2$ & $1.8 \pm 10.7$ & $19.26 \pm 4.41$ & $4.3 \pm 9.5^{*}$ & $2.1 \pm 10.1$ \\
\hline \multirow[t]{8}{*}{ Femoral shaft } & Cortical thickness (mm) & $3.71 \pm 0.62$ & $0.7 \pm 5.1$ & $2.6 \pm 4.5^{*}$ & $3.91 \pm 0.62$ & $-0.7 \pm 4.6$ & $-1.3 \pm 3.9$ \\
\hline & Cortical CSA $\left(\mathrm{cm}^{2}\right)$ & $2.22 \pm 0.39$ & $1.7 \pm 5.2$ & $2.7 \pm 3.6^{*}$ & $2.35 \pm 0.39$ & $-0.6 \pm 4.1$ & $-0.5 \pm 3.0$ \\
\hline & Total CSA $\left(\mathrm{cm}^{2}\right)$ & $2.38 \pm 0.38$ & $1.7 \pm 5.0$ & $2.5 \pm 3.4^{*}$ & $2.5 \pm 0.39$ & $-0.5 \pm 4.0$ & $-0.1 \pm 3.0$ \\
\hline & Cortical perimeter $(\mathrm{cm})$ & $10.27 \pm 0.6$ & $0.4 \pm 3.8$ & $-0.7 \pm 2.5$ & $10.3 \pm 0.7$ & $0.2 \pm 4.3$ & $0.5 \pm 3.2$ \\
\hline & Cortical vBMD $\left(\mathrm{mg} / \mathrm{cm}^{3}\right)$ & $879.65 \pm 70.77$ & $0.4 \pm 2.7$ & $0.1 \pm 3.6$ & $892.97 \pm 59.03$ & $0.3 \pm 4.1$ & $-0.9 \pm 3.1$ \\
\hline & Total vBMD $\left(\mathrm{mg} / \mathrm{cm}^{3}\right)$ & $461.36 \pm 77.37$ & $0.7 \pm 5.1$ & $1.1 \pm 5.7$ & $482.05 \pm 74.95$ & $-0.2 \pm 5.2$ & $-1.4 \pm 4.3$ \\
\hline & $\mathrm{SM}\left(\mathrm{cm}^{3}\right)$ & $0.88 \pm 0.18$ & $1.3 \pm 5.9$ & $2.7 \pm 7.2$ & $0.93 \pm 0.2$ & $-0.8 \pm 5.2$ & $0.3 \pm 4.8$ \\
\hline & $\mathrm{BR}$ & $3.67 \pm 0.88$ & $-0.4 \pm 7.7$ & $-3.3 \pm 5.4^{*}$ & $3.39 \pm 0.75$ & $0.9 \pm 6.7$ & $1.9 \pm 5.3$ \\
\hline
\end{tabular}

Data are mean $\pm \mathrm{SD}$

$Q C T$ quantitated computed tomography, $C S A$ cross-sectional area, $v B M D$ volumetric bone mineral density, $S M$ section modulus, $B R$ buckling ratio ${ }^{*} p<0.05 ;{ }^{* *} p<0.01$ compared with baseline

thickness and those in the other parameters at the femoral neck at 72 weeks were analyzed, since cortical thickness was most significantly improved following once-weekly teriparatide treatment. Percent changes in cortical thickness at the femoral neck had significant positive correlations with percent change of cortical CSA $(r=0.612, p<0.0001)$, total CSA $(r=0.389$, $p=0.0062)$, total vBMD $(r=0.546, p<0.0001)$, and SM $(r=$ $0.523, p=0.0001)$ in the teriparatide group. Negative correlations were found between percent changes of cortical thickness and cortical perimeter $(r=-0.561, p<0.0001)$ or BR $(r=$ $-0.905, p<0.0001)$ in teriparatide group. The same trends in the correlation between cortical thickness and the other parameters were observed in placebo group. The correlation between percent change in cortical thickness and BR at the femoral neck was higher in the teriparatide group $\left(r^{2}=0.82\right)$ than in the placebo group $\left(r^{2}=0.54\right)$. There was no significant correlation between the percent change in cortical thickness and that of cortical vBMD in either group.
To visualize the relationships of multiple parameters at the individual level, the percent change in cortical thickness at the femoral neck was plotted on the horizontal axis of each panel in Fig. 4 versus the percent changes in cortical CSA (Fig. 4a), perimeter (Fig. 4b), SM (Fig. 4c), and BR (Fig. 4d), separately for the teriparatide (solid lines) and placebo (dashed lines) groups. Each panel of Fig. 4 is divided into four quadrants and the percentages of closed circles (teriparatide) and open circles (placebo) included in each quadrant are provided in the figure. The linear regression lines are basically the same between the teriparatide and placebo groups. Further, with respect to parameters with positive correlations (Fig. 4a, c), the distribution of individual data in the teriparatide group is significantly different from placebo (cortical CSA: $p=0.0111$, SM: $p=$ 0.0250); weighted distribution of closed circles (teriparatide) in the first quadrant is high, while the open circles (placebo) are highly distributed in the third quadrant. Similarly, in the case of parameters with negative correlations (Fig. 4b, d), the 
a
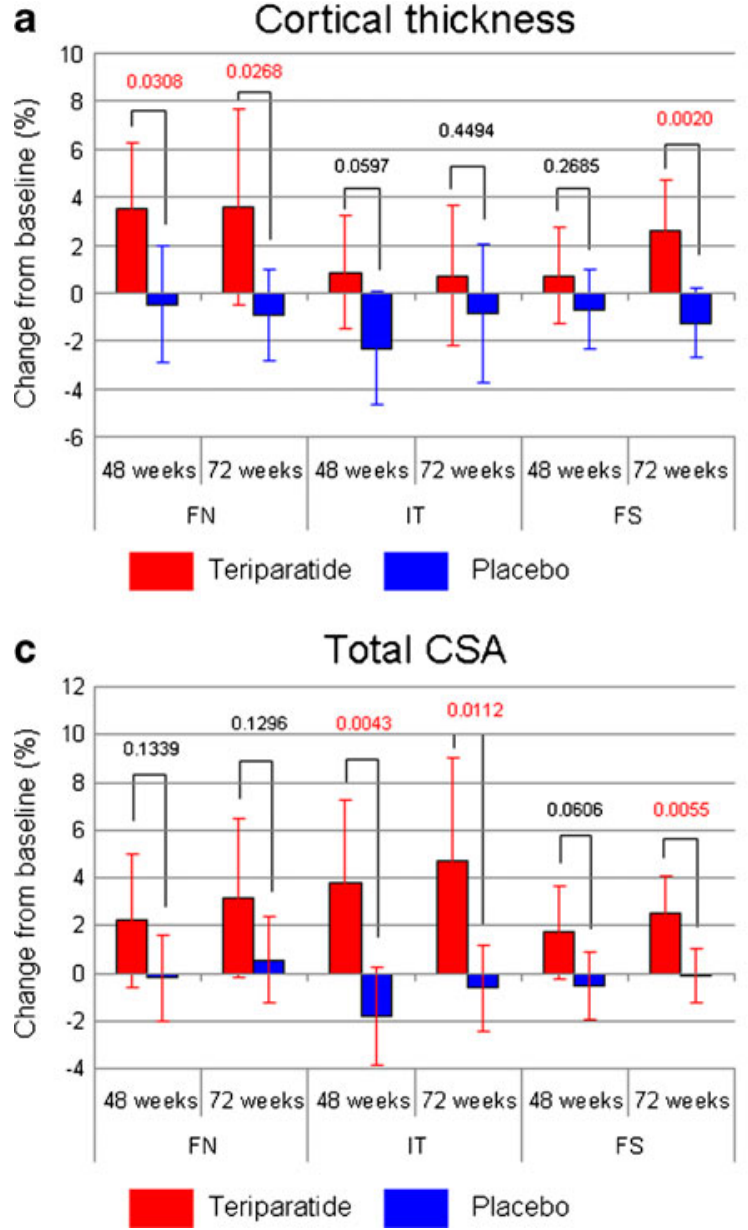

Fig. 1 Mean percent changes and $95 \%$ confidence interval from baseline in cortical thickness (a), cortical cross-sectional area (CSA) (b), total CSA (c), and cortical perimeter (d) at 48 and 72 weeks of treatment with teriparatide and placebo. Changes at the femoral neck $(F N)$, inter-trochanter (IT), and femoral shaft (FS) are shown. Values on top of each
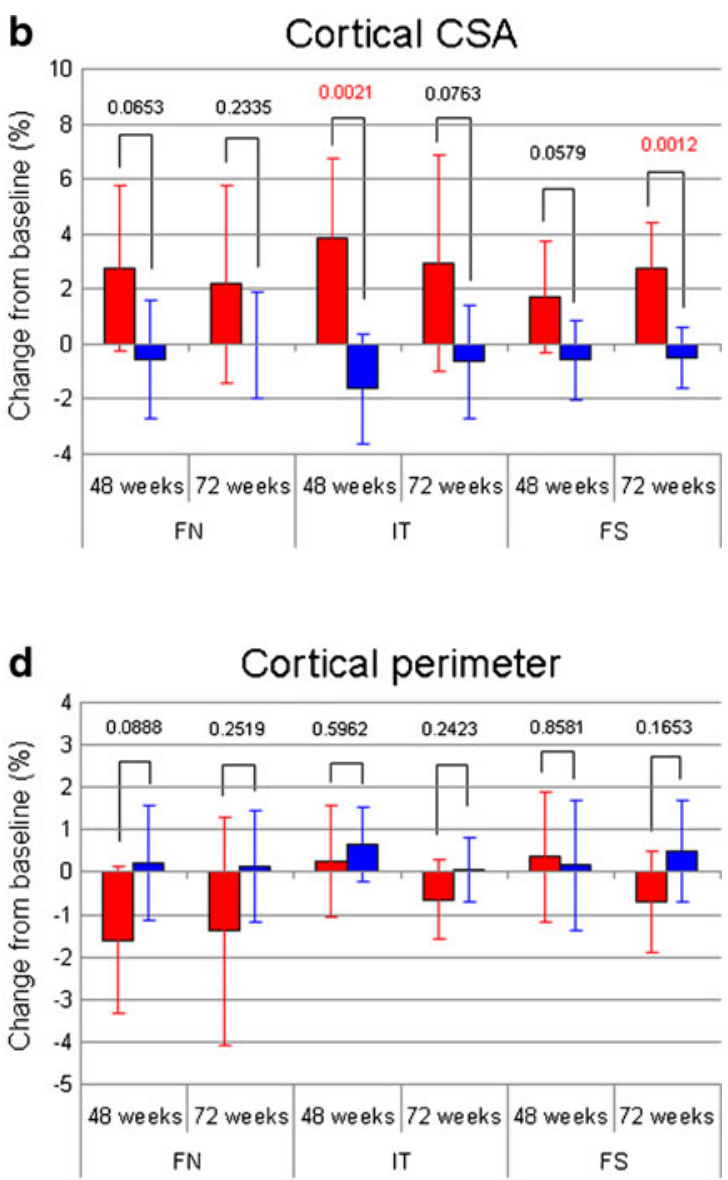

panel indicate $p$ values (between teriparatide and placebo group). Red and blue bars correspond to teriparatide and placebo groups, respectively. To compare the difference between the two groups, the percent changes from baseline in QCT parameters were analyzed using the Student's $t$ test

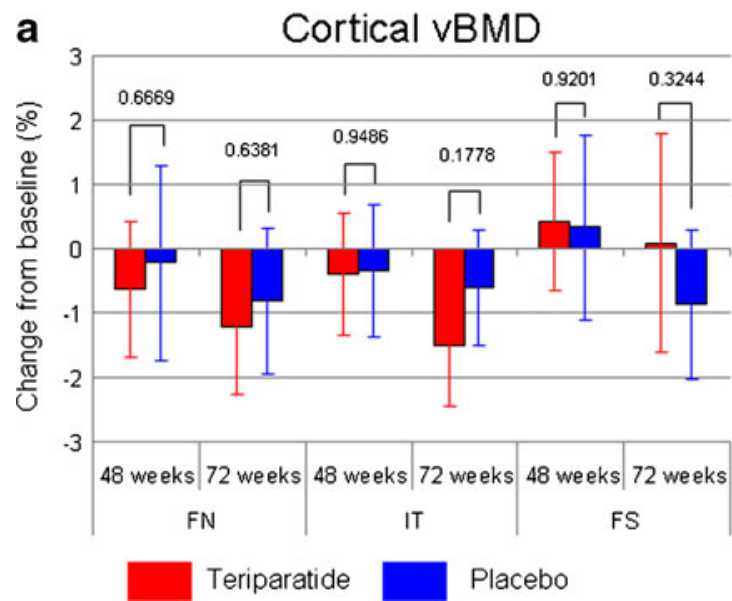

Fig. 2 Mean percent changes and $95 \%$ confidence interval from baseline in cortical volumetric bone mineral density (vBMD) (a) and total vBMD (b) at 48 and 72 weeks of treatment with teriparatide and placebo. Changes at the femoral neck $(F N)$, inter-trochanter $(I T)$, and femoral shaft $(F S)$ are shown. Values on top of each panel indicate $p$ values

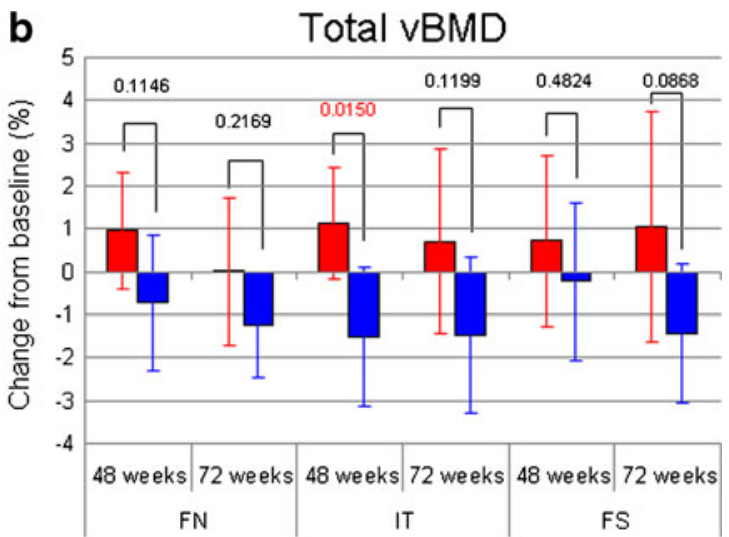

(between teriparatide and placebo group). Red and blue bars correspond to teriparatide and placebo groups, respectively. To compare the difference between the two groups, the percent changes from baseline in QCT parameters were analyzed using the Student's $t$ test 


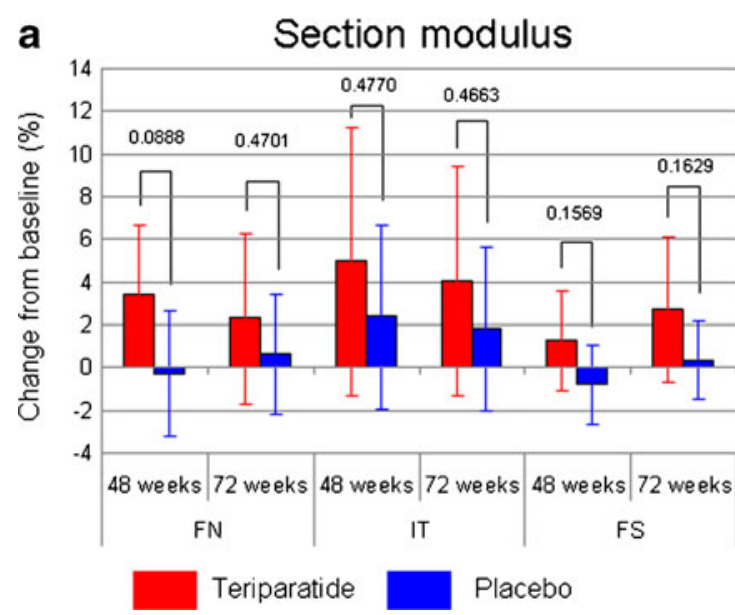

Fig. 3 Mean percent changes and $95 \%$ confidence interval from baseline in SM (a) and BR (b) at 48 and 72 weeks of treatment with teriparatide and placebo. Changes at the femoral neck $(F N)$, inter-trochanter $(I T)$, and femoral shaft $(F S)$ are shown. Values on top of each panel indicate

distribution of closed circles (teriparatide) in the fourth quadrant is high, while the open circles (placebo) are highly distributed in the second quadrant. The difference between teriparatide and placebo is significant for BR $(p=0.0274)$. These results suggest that changes in the placebo group with natural aging (i.e., age-related deteriorations in proximal femur geometry and biomechanical properties) are reversed at least partially by once-weekly teriparatide treatment.

\section{Discussion}

This longitudinal assessment by CT demonstrates the changes in bone geometry, $\mathrm{vBMD}$, and mechanical properties at the proximal femur by once-weekly injection of $56.5 \mu \mathrm{g}$ teriparatide for 72 weeks. This is the first longitudinal CT study to include comparison with a double-blinded placebo group. Previous studies have evaluated the effects of teriparatide on proximal femur geometry and its biomechanical properties using CT [8], but they did not include a placebo group.

Generally, the effects of once-weekly teriparatide injection on proximal femur geometry in this study are similar to results with daily teriparatide injections reported in a subgroup of the EUROFORS study (EU-CT study) [8]. The same analysis software program was employed and the main effects included increases in cortical thickness/CSA as well as total vBMD. Cortical thickness/CSA increasing while bone perimeter remained unchanged over 72 weeks of once-weekly teriparatide, suggests that cortical bone formation took place at the endosteal surface resulting in an increase in cortical thickness with a significant decrease in BR.

One difference observed between the weekly and daily treatment regimens is the effect on cortical vBMD. Although only eight patients were included in the treatment-naïve group

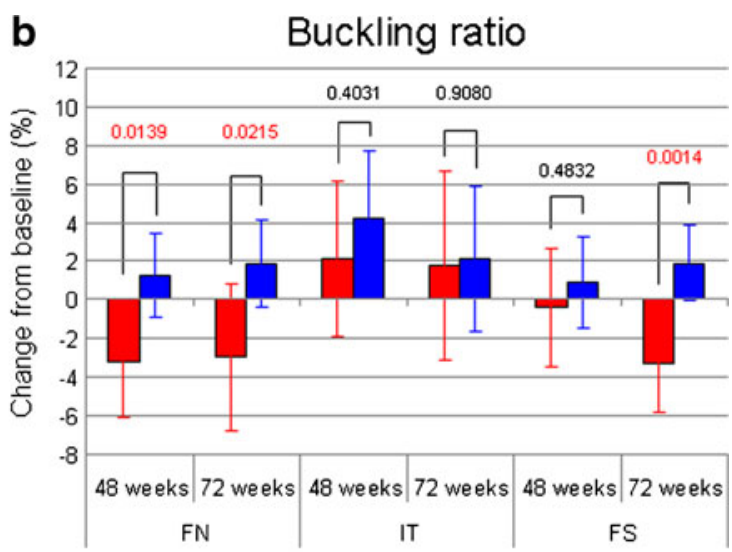

$p$ values (between teriparatide and placebo group). Red and blue bars correspond to teriparatide and placebo groups, respectively. To compare the difference between the two groups, the percent changes from baseline in QCT parameters were analyzed using the Student's $t$ test

in the EU-CT study, daily teriparatide decreased cortical vBMD at the femoral neck after 6 months of treatment ( $\sim 3.0 \%$ from baseline), which was consistent with the results of a previous large clinical trial [11]. Moreover, a decrease in cortical BMD at the femoral neck with 12 months of daily teriparatide treatment [12] and a decrease in cortical BMD at the distal radius and tibia were reported [13]. In contrast, our results showed that once-weekly teriparatide maintained cortical vBMD at the femoral neck $(-0.6 \%, 48$ weeks and $-1.2 \%, 72$ weeks). This difference may be due to distinct patterns of bone remodeling between daily and weekly teriparatide treatment given that weekly teriparatide caused an increase in serum osteocalcin (bone formation marker) and a decrease in urinary NTX (bone resorption marker) [5]. Other factors such as cohort effects, differences in $\mathrm{CT}$ acquisition or the software may also have had an effect and help to explain the differences.

The question of whether or not teriparatide stimulates periosteal apposition has been raised. In the EU-CT study, total CSA (cortical CSA plus marrow area) did not change significantly throughout the study period and the authors concluded that there was no detectable periosteal apposition. DXA-based hip structure analysis (HSA), conducted as a subgroup of the Fracture Prevention Trial (DXA-HSA study) [9], also showed that periosteal apposition appeared to be reduced in patients receiving daily teriparatide in comparison with a placebotreated group. On the other hand, some studies reported daily treatment with teriparatide seemed to stimulate new bone formation on the periosteal and endosteal surfaces $[14,15]$. Thus, periosteal and endosteal apposition may be stimulated within a certain time window or may vary depending on skeletal sites, such as weight bearing or non-weight bearing bone [13].

Bone generally expands in diameter with age $[16,17]$, as less bone density requires a wider bone to maintain bending strength. It has been speculated that expansion is a 
a

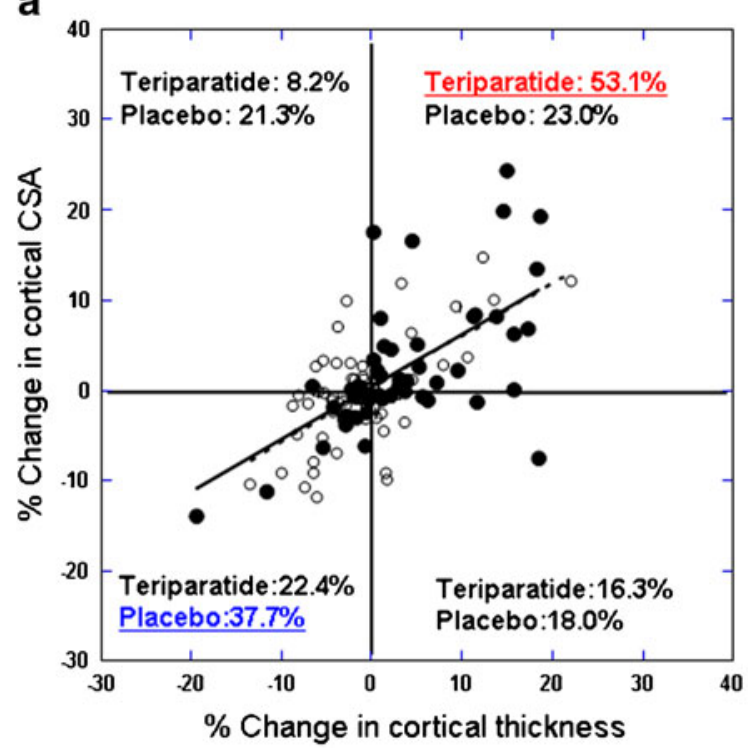

C

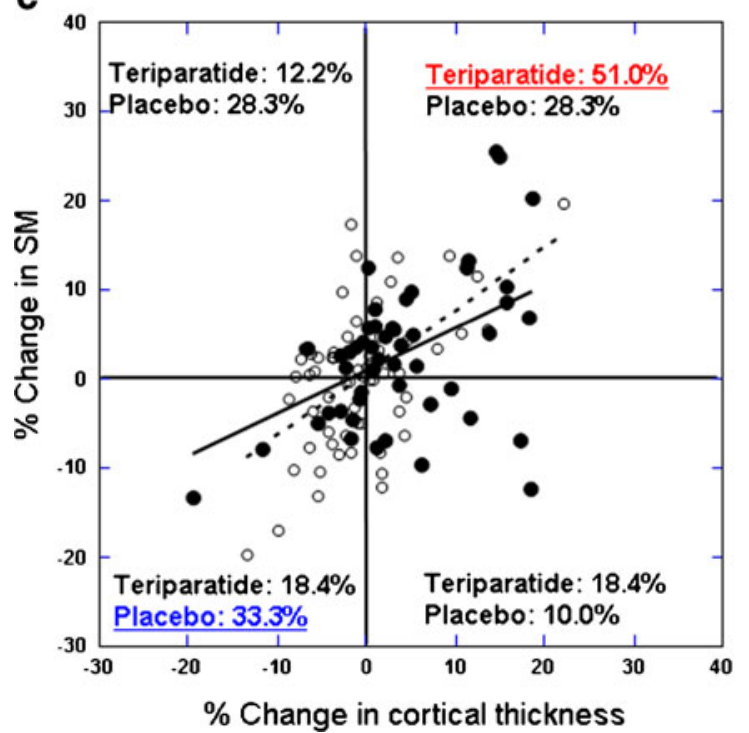

Fig. 4 Weekly administration of teriparatide reverses age-related changes at 72 weeks in cortical geometry and biomechanical properties at the femoral neck. Relationships between percent changes in cortical thickness versus those in cortical cross-sectional area $(C S A)(\mathbf{a})$, perimeter $(\mathbf{b})$, SM (c), or BR (d) are shown. Solid circles and open circles correspond to percent changes of individuals in the teriparatide and placebo groups, respectively. Note that linear regression lines for teriparatide (solid lines) and placebo (dashed lines) showing the relationship between the percent change in cortical thickness and those in other parameters, are almost identical regardless of whether the correlation is positive (a and $\mathbf{c}$ ) or

homeostatic adaptation to a net bone loss in order to maintain bone strength $[18,19]$. This age-related adaptive response was not seen in the placebo group of the current study. Onceweekly injection of teriparatide increased cortical thickness with no change in cortical perimeter at the femoral neck. Thus, it is tempting to speculate that as a result of increased cortical thickness (which improves bone strength), periosteal apposition may not be required under once-weekly teriparatide b

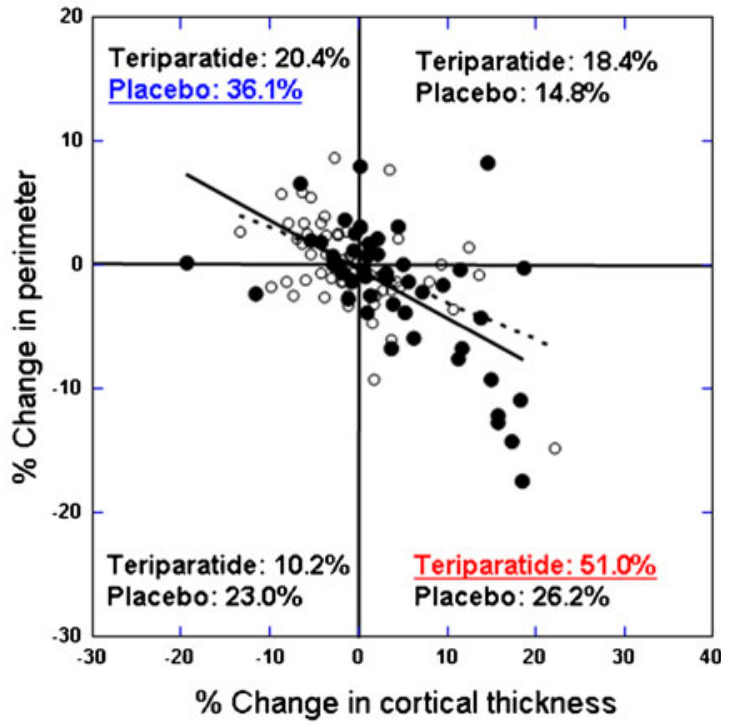

d

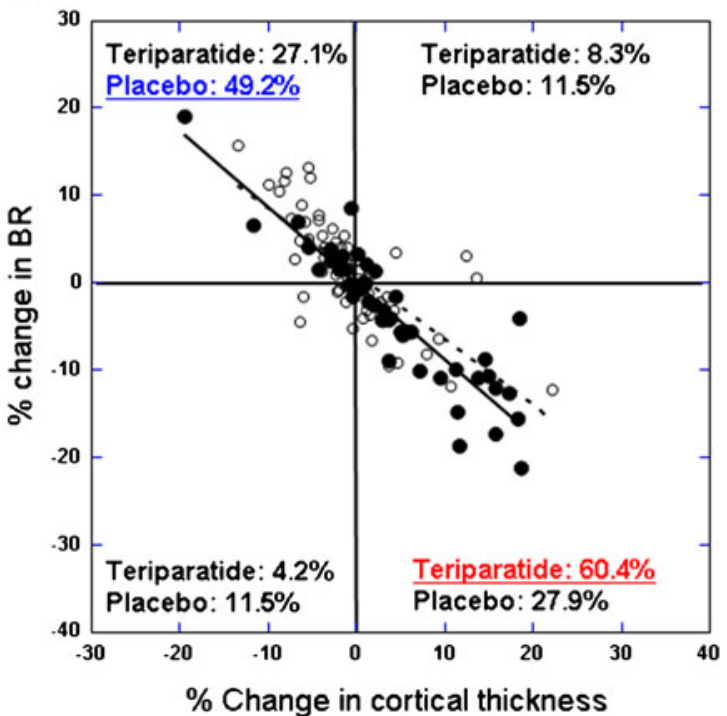

negative (b and $\mathbf{d})$. The distribution of closed and open circles in each quadrant is shown in percentages. Note that with respect to the parameters with positive correlations (a and $\mathbf{c}$ ), the relative distribution of open circles (for placebo) in the third quadrant is shifted to the first quadrant by weekly teriparatide (closed circles). Similarly, in the case of the parameters with negative correlations (b and $\mathbf{d}$ ), relative distribution of open circles (for placebo) in the second quadrant is shifted to the fourth quadrant by weekly teriparatide (closed circles), suggesting that weekly teriparatide reversed age-related changes in proximal femur geometry and biomechanical properties

treatment. Actually, a change in BR based upon improvement in cortical thickness was observed in the teriparatide group. The $r^{2}$ between percent change of cortical thickness and that of $\mathrm{BR}$ in the teriparatide group was higher than the placebo group.

As illustrated in Fig. 4, teriparatide improved all geometry and biomechanical parameters, while maintaining their relationships with changes in cortical thickness (as in the placebo 
group). However, the distribution patterns of their relationships indicate that the effect of teriparatide is in the exact opposite direction of age-related skeletal changes. It is suggested, therefore, that compared with the changes in the placebo group, once-weekly teriparatide injection reverses age-related deteriorations in bone structure and strength by increasing cortical thickness/CSA and total vBMD, not increasing cortical perimeter, and improving biomechanical parameters.

In our previous study which characterized femoral neck geometry in patients with hip versus trochanteric fractures and compared them with age-matched controls [7], patients with femoral neck fracture had a significantly longer hip axis length (HAL), lower cross-sectional moment of inertia (CSMI), and higher BR, while those with trochanteric fractures had a smaller cortical CSA of the femoral neck. Once-weekly teriparatide may improve all these geometric changes. Taken together with the present results that SM (calculated as CSMI) divided by the maximal distance to center of the bone mass (centroid) increased by approximately $4 \%$, and BR decreased approximately $4 \%$ compared to placebo, once-weekly injection of $56.5 \mu \mathrm{g}$ teriparatide may have the potential to reduce the risk of hip fracture.

In the current longitudinal study, we also analyzed the geometry and biomechanical properties at the intertrochanter and shaft regions in addition to those at the femoral neck. The percent changes in several parameters at the femoral neck and inter-trochanter were greater at 48 weeks compared to 72 weeks, while at the femoral shaft, the changes were greater at 72 weeks compared to 48 weeks, suggesting that the effects of teriparatide at the shaft take place in a later phase than those at the femoral neck and inter-trochanter. Endosteal bone formation might appear later at the purely cortical site, such as femoral shaft. Similar results were observed in the DXA-HSA study [9], in which teriparatide seemed to have no significant effects on femoral shaft geometrical parameters.

A limitation of our study was the small number of subjects; since all the participating institutes in the TOWER trial were not equipped with MDCT scanners, the number of subjects with CT scans was limited. We paid careful attention, for example, to the CT images and those with artifacts were excluded from the study. However, the results of this study were proved by comparison with the placebo group. Another limitation was that we had no confirmation on the event of hip fracture, since no new hip fracture was reported in either group. As an additional limitation, Mindways software was used for analyzing the geometry of inner and outer surfaces of the cortex and this method may not currently be the best available technology for this evaluation. However, we carefully applied this program to define the same region of an individual subject for analysis, using the "Optimize FN Axis" algorithm. When this algorithm did not work well and different regions were obtained, we carefully manually adjusted both the axis of the femoral neck and the axis of the femoral shaft, visually comparing the baseline CT image and the treatment image. In addition, we improved the reproducibility using the eccentricity registration method for measurement of the femoral neck.

In conclusion, we have demonstrated (using $\mathrm{CT}$ and 3D analysis) that once-weekly teriparatide increased cortical thickness and cortical and total CSA, and improved biomechanical indices. Moreover, once-weekly teriparatide did not increase cortical perimeter but seemed to effectively reverse changes in proximal femur geometry with aging. Taken together with its anti-fracture efficacy in the spine [5], onceweekly $56.5 \mu \mathrm{g}$ teriparatide administration may have the potential to prevent hip fracture.

Acknowledgments This study was jointly designed by all authors and the sponsor (Asahi Kasei Pharma Corporation). The sponsor takes responsibility for data collection and quality control. Analyses for publication were the joint responsibility of the all author and the sponsor. All authors contributed to the manuscript and agreed on the final version. We thank the doctors who participated in the clinical trial.

Conflicts of interest Funding for this study was supported by the Asahi Kasei Pharma Corporation. This study was also supported in part by a grant for the Promotion of Fundamental Studies in Health Sciences from the National Institute of Biomedical Innovation (NIBIO) of Japan (Grant \#06-31 to MI). MI has received research grants and consulting fees or other remuneration from Chugai, Daiichi Sankyo, Ono Pharmaceutical Company, and Asahi Kasei Pharma. RO is an employee of Asahi Kasei Pharma Corporation. MF has received consulting fees from Asteras and Asahi Kasei Pharma. TSu has received research grants and consulting fees from the pharmaceutical companies Asahi Kasei Pharma and Daiichi Sankyo. MS has received consulting fees from the pharmaceutical companies Asahi Kasei Pharma, Dai-ichi Sankyo, Chugai, and Teijin Pharma. TN has received research grants and/or consulting fees from the pharmaceutical companies Chugai, Teijin, Asahi Kasei Pharma, and Dai-ichi Sankyo. TN is a counselor for hospital administration and social medical insurance with the Japan Ministry of Health, Welfare, and Labour. TSo and YN declare that they have no conflicts of interest.

Open Access This article is distributed under the terms of the Creative Commons Attribution Noncommercial License which permits any noncommercial use, distribution, and reproduction in any medium, provided the original author(s) and the source are credited.

\section{References}

1. Podbesek R, Edouard C, Meunier PJ, Parsons JA, Reeve J, Stevenson RW, Zanelli JM (1983) Effects of two treatment regimens with synthetic human parathyroid hormone fragment on bone formation and the tissue balance of trabecular bone in greyhounds. Endocrinology 112:1000-1006

2. Hock JM, Gera I (1992) Effects of continuous and intermittent administration and inhibition of resorption on the anabolic response of bone to parathyroid hormone. J Bone Miner Res 7:65-72

3. Neer RM, Arnaud CD, Zanchetta JR, Prince R, Gaich GA, Reginster J-Y, Hodsman AB, Eriksen EF, Ish-Shalom S, Genant HK, Wang O, Mitlak BH (2001) Effect of parathyroid hormone (1-34) on fractures 
and bone mineral density in postmenopausal women with osteoporosis. N Engl J Med 344:1434-1441

4. Fujita $T$, Inoue $T$, Morii $H$, Morita R, Norimatsu $H$, Orimo $H$, Takahashi HE, Yamamoto K, Fukunaga M (1999) Effect of an intermittent weekly dose of human parathyroid hormone (1-34) on osteoporosis: a randomized double-masked prospective study using three dose levels. Osteoporos Int 9:296-306

5. Nakamura T, Sugimoto T, Nakano T, Kishimoto H, Ito M, Fukunaga M, Hagino H, Sone T, Yoshikawa H, Nishizawa Y, Fujita T, Shiraki M (2012) Randomized Teriparatide [human parathyroid hormone (PTH) 1-34] Once-Weekly Efficacy Research (TOWER) trial for examining the reduction in new vertebral fractures in subjects with primary osteoporosis and high fracture risk. J Clin Endocrinol Metab 97:3097-3106

6. Ito M, Nakata T, Nishida A, Uetani M (2011) Age-related changes in bone density, geometry and biomechanical properties of the proximal femur: CT-based 3D hip structure analysis in normal postmenopausal women. Bone 48:627-630

7. Ito M, Wakao N, Hida T, Matsui $\mathrm{Y}$, Abe $\mathrm{Y}$, Aoyagi K, Uetani M, Harada A (2010) Analysis of hip geometry by clinical CT for the assessment of hip fracture risk in elderly Japanese women. Bone 46: 453-457

8. Borggrefe J, Graeff C, Nickelsen TN, Marin F, Gluer CC (2010) Quantitative computed tomographic assessment of the effects of 24 months of teriparatide treatment on 3D femoral neck bone distribution, geometry, and bone strength: results from the EUROFORS study. JBMR 25:472-481

9. Uusi-Rasi K, Semanick LM, Zanchetta JR, Bogado CE, Eriksen EF, Sato M, Beck TJ (2005) Effects of teriparatide [rhPTH(1-34)] treatment on structural geometry of the proximal femur in elderly osteoporotic women. Bone 36:948-958

10. Ito M, Nakamura T, Fukunaga M, Shiraki M, Matsumoto T (2011) Effect of eldecalcitol, an active vitamin D analog, on hip structure and biomechanical properties: 3D assessment by clinical CT. Bone 49: $328-334$
11. Black DM, Greenspan SL, Ensrud KE, Palermo L, McGowan JA, Lang TF, Garnero P, Bouxsein ML, Bilezikian JP, Rosen CJ, for the PaTH Study Investigators (2003) The effects of parathyroid hormone and alendronate alone or in combination in postmenopausal osteoporosis. N Engl J Med 349:1207-1215

12. Keaveny TM, Hoffmann PF, Singh M, Palermo L, Bilezikian JP, Greenspan SL, Black DM (2008) Femoral bone strength and its relation to cortical and trabecular changes after treatment with $\mathrm{PTH}$, alendronate, and their combination as assessed by finite element analysis of quantitative CT scans. J Bone Miner Res 23:1974-1982

13. Macdonald HM, Nishiyama KK, Hanley DA, Boyd SK (2011) Changes in trabecular and cortical bone microarchitecture at peripheral sites associated with 18 months of teriparatide therapy in postmenopausal women with osteoporosis. Osteoporos Int 22:357-362

14. Lindsay R, Zhou H, Cosman F, Nieves J, Dempster DW, Hodsman AB (2007) Effects of a one-month treatment with PTH(1-34) on bone formation on cancellous, endocortical, and periosteal surfaces of the human ilium. J Bone Miner Res 22:495-502

15. Zanchetta JR, Bogado CE, Ferretti JL, Wang O, Wilson MG, Sato M, Gaich GA, Dalsky GP, Myers SL (2003) Effects of teriparatide [recombinant human parathyroid hormone (1-34)] on cortical bone in postmenopausal women with osteoporosis. J Bone Miner Res 18: $539-543$

16. Seeman E (2002) Pathogenesis of bone fragility in women and men. Lancet 359:1841-1850

17. Seeman E (2003) Periosteal bone formation-a neglected determinant of bone strength. N Engl J Med 349:320-323

18. Beck TJ, Oreskovic TL, Stone KL, Ruff CB, Ensrud K, Nevitt MC, Genant HK, Cummings SR (2001) Structural adaptation to changing skeletal load in the progression toward hip fragility: the study of osteoporotic fractures. J Bone Miner Res 16:1108-1119

19. Feik SA, Thomas CD, Bruns R, Clement JG (2000) Regional variations in cortical modeling in the femoral mid-shaft: sex and age differences. Am J Phys Anthropol 112:191-205 